\title{
DEFINICIÓN DE “AGRICULTURA FAMILIAR” COMO CATEGORÍA SOCIOECONÓMICA
}

\section{DEFINIÇÃO DE “AGRICULTURA FAMILIAR” COMO CATEGORIA ECONÔMICA}

\author{
Álvaro Ramos \\ FIDA MERCOSUR - Montevideo - Uruguay
}

\begin{abstract}
Resumen: El objeto del presente artículo es definir consistentemente y con claridad qué características son propias definen a la "Agricultura familiar". De esta forma se realizó una recopilación de las definiciones y metodologías comúnmente más aceptadas y utilizadas a nivel de los países, así como a nivel regional e internacional para definir y caracterizar a la agricultura familiar, tomando en cuenta su diversidad. Además o estudio intenta hacer definiciones sobre la AF como categoría socioeconómica de análisis y como las dichas definiciones se sustenten en criterios y luego en parámetros e indicadores, que puedan hacer comparables situaciones muy diferentes. El desafío ahora es reconocer la heterogenidad de la AF y comenzar a hablar de "matrices de políticas públicas" que permitan atender la complejidad de la finca familiar, la necesidad de asociar las mismas para asegurar el autoconsumo familiar y competir, así como la necesidad de asegurar los parámetros de la SAN. Finalmente, encarar una análisis de la AF como categoría socio / económica no es atemporal ni a - geográfica, está fuertemente vinculada a cada contexto y cada contexto tiene que ver con estos factores: (a) los derivados de la situación geográfica y agroecológica y distancia relativa a los mercados; (b) los derivados de los procesos económicos; (c) los derivados de la historia, la cultura, la relación con la tierra y el territorio. El Concepto de AF es una construcción multidimensional. Dentro de ella los criterios fundamentales son: (i) organización del trabajo familiar en la finca y contratación eventual de mano de obra asalariada; (ii) administración de la finca o empresa familiar, o sea quién toma las decisiones productivas y/o técnicas y/o comerciales; (c) situación relativa de autonomía o dependencia respecto de los distintos mercados (insumos, factores, productos).

Palabras-llave: El desarrollo rural. Definición teórica. Agricultura.
\end{abstract}

Resumo: O objetivo deste artigo é definir, claramente, quais recursos são característicos do conceito de agricultura familiar - AF. Assim, realizou-se uma compilação das mais comuns, aceitas e utilizadas definições em nível de país. A metodologia utilizada considerou os níveis regional e internacional para definir e caracterizar a agricultura familiar, levando em conta a sua diversidade. Além disso, o estudo busca definições de AF como uma categoria socioeconômica de análise nas quais as definições são sustentadas por critérios e, seguidas de parâmetros e de indicadores que permitam a utilização de forma comparável em diferentes situações. O desafio é reconhecer a heterogeneidade da AF e começar a utilizar "matrizes de ordem pública" que abordam a complexidade da propriedade familiar, a necessidade de associá-las para garantir o consumo familiar e a concorrência, bem como a necessidade de garantir parâmetros de SAN. Finalmente, enfrenta-se uma análise da $\mathrm{AF}$ como uma categoria econômica não atemporal ou geográfica, mas fortemente ligada a cada contexto que deve derivar dos seguintes fatores: (a) situação geográfica e agroecológica e distância nos mercados; (B) processos econômicos; (C) história, cultura, relação com a terra e território. O conceito AF é um constructo multidimensional e dentro dele há critérios fundamentais, a saber: (i) a organização do trabalho familiar na propriedade e eventual de contratação; (Ii) a administração da propriedade pela família, que é quem tomar decisões técnicas produtivas e/ou comerciais; (C) situação relativa de autonomia ou dependência de mercados individuais (entradas, fatores, produtos).

Palavras-chave: Desenvolvimento rural. Definição teórica. Agricultura. 


\section{Introducción y antecedentes}

El presente artículo fue preparado a partir de los aspectos sustantivos que justificaron y orientaron un trabajo de investigación realizado por el autor, coordinando un "panel de expertos" liderado por FAO en el año 2014 (Año de la Agricultura Familiar) y que tuvo como cometido llegar a una definición suficientemente amplia, pero a la vez precisa, que permitiera definir consistentemente y con claridad qué características son propias definen a la "Agricultura familiar". A la vez que sistematizar el conjunto de sub categorías que hacen parte de un número tan amplio y heterogéneo de unidades productivas que son las responsables por la producción del orden del $70 \%$ de los alimentos integrantes de las canastas básicas de seguridad alimentaria, en la mayoría de los países del mundo.

De esta forma se realizó una recopilación de las definiciones y metodologías comúnmente más aceptadas y utilizadas a nivel de los países, así como a nivel regional e internacional para definir y caracterizar a la agricultura familiar, tomando en cuenta su diversidad.

El trabajo del Panel de expertos convocado y liderado por la FAO se dedicó a generar y sistematizar la información, criterios y parámetros para llegar a una conceptualización general de la AF como categoría de análisis socio / económica y productiva.

El objetivo no fue exclusivamente teórico o académico, sino que puso énfasis y se sustentó en el criterio que, una más precisa definición de "Agricultura Familiar" como categoría de análisis general y de las emergentes sub - categorías, permitirían focalizar mejor las políticas públicas diferenciadas, articular estrategias, diseñar políticas e instrumentos y asignar recursos hacia aquellas inversiones claves para la producción de bienes y servicios públicos. Así mismo construir infraestructura adaptada y localizada. Generar innovaciones C\&T necesarias para aumentar la productividad de la AF, aumentar su competitividad relativa en los mercados, favorecer su inserción en éstos y en cadenas de valor inclusivas. Al mismo tiempo atender las necesidades derivadas de su situación económico / financiera, superar la pobreza - cuando necesario - y en generar mejorar sostenidamente la calidad de vida de las familias dedicadas a esta actividad.

El Panel no se dedicó exclusivamente a realizar una investigación académica respaldada por toda la evidencia empírica posible consultar, sino que encaró el debate e intercambio de información con un enfoque pragmático, buscando: (i) por un lado definiciones sobre la AF como categoría socioeconómica de análisis; (ii) que dichas definiciones se sustenten en criterios y luego en parámetros e indicadores, que puedan hacer comparables situaciones muy diferentes.

La heterogeneidad intrínseca de la AF como categoría de análisis se basa en: (a) el tipo de población rural que involucra; (b) por el tipo de organización del trabajo y la producción en las fincas; (c) por las respuestas a las situaciones agroecológicas de los territorios (áreas geográficas) en las cuales se afincan; (d) por su relación con los mercados y otros agentes económicos y actores sociales.

El trabajo realizado se trató de una investigación amplia, por la cobertura geográfica y de realidades relativas a niveles regionales y sub - regionales, y de carácter instrumental. Buscó generar antecedentes y una orientación metodológica para dotar de instrumentos de análisis y categorización, para mejor focalizar políticas, diseñar instrumentos y alocar recursos. 
El Panel de expertos estimuló un debate a nivel internacional y regional que permitió, entre otras cosas en valorar la evolución en los últimos años de la "visibilidad" pública y política de la AF, y el impacto de esta visibilidad sobre las características, calidad y sostenibilidad de las políticas públicas diferenciadas y sobre la institucionalidad que las aplica.

Esta realidad no es nada menor, pues la forma de aproximación por parte de las autoridades nacionales en el diseño y aplicación de las políticas sectoriales agrícolas, ganaderas, forestales de pesca y/o piscicultura, sufrió un cambio sustantivo, evolucionando - en el caso de la AF - desde las políticas verticales por rubro o sub - sector de la producción agrícola, por un lado y por otro políticas transversales relativamente aisladas o divorciadas de las primeras, hasta llegar a un concepto sistémico que requiere, a partir de un trabajo interdisciplinario y acciones interinstitucionales, diseñar y aplicar el concepto de "matrices de políticas" que se aplican sobre un territorio dado. El concepto de territorio es el de una construcción social, sobre una plataforma física y agroecológica, donde la AF dentro de la población rural - es un factor clave para los procesos de desarrollo.

Durante los debates se encontró un claro consenso, más allá de las particularidades de cada contexto, regional, sub - regional o nacional, sobre la necesidad de entender que en este tipo de políticas y de intervenciones hay que combinar y esperar resultados de procesos: (a) biológicos; (b) económicos, (c) sociales y (d) culturales.

Se buscó llegar a consensuar un "concepto paraguas" sobre la AF para trabajar con criterios similares para las diferentes regiones y países del mundo y cuantificar y representar las realidades particulares y la heterogeneidad de la categoría de la que estamos hablando, mediante parámetros ligados a cada uno de los criterios. Alcanzados estos objetivos será posible además incorporar dichos criterios y parámetros a los instrumentos estadísticos de recolección de datos (censos / registros), elementos indispensable para disponer de una información cada vez más fiel de situaciones y características de la AF, no solo desde el punto de vista productivo / económico, sino también desde el punto de vista de las otras áreas del conocimiento que permiten caracterizar y representar a la AF.

Obviamente el trabajo encontró diversas definiciones en relación a: (i) las regiones geográficas; (ii) las pautas culturales o ideológicas implícitas en el acercamiento al tema; (iii) definiciones de tipo académico, necesarias para los trabajos de investigación técnico / científicos; (iv) definiciones referidas a políticas públicas, con o sin sustento jurídico; (v) definiciones basadas en criterios de representatividad utilizadas por organizaciones sociales de la AF.

\section{Validez del esfuerzo para encontrar una definición consistente de "Agricultura Familiar"}

Desde los años 2003/2004 y hasta el momento actual, ha crecido en importancia la visibilidad pública y política de la AF, tanto en los países de nuestra región (MERCOSUR ampliado), de ALC y en general, así ocurrió también en el mundo, especialmente a partir de la campaña para designar al año 2014 como "Año de la Agricultura Familiar" por la Asamblea General de las Naciones Unidas.

Las Políticas Públicas Diferenciadas para la AF, fueron asumidas en los últimos 15/17 años como una parte estratégica de las políticas de desarrollo, crecimiento económico, justicia social y redistributiva de una gran mayoría de países en el mundo, en todos los continentes y regiones. A la vez que grandes conglomerados económicos de 
países desarrollados como los de la Unión Europea siempre la tuvieron presente, con otros nombres ciertamente, como el de Política Agrícola Comunitaria (PAC), dentro de las estrategias de seguridad y aún soberanía alimentaria de sus estados nacionales.

El aumento de la visibilidad pública y política para la AF, así como la generalización de un abordaje de políticas diferenciadas para la AF por parte de los diferentes Estados y Agencias multilaterales de Cooperación, encontró una realidad académica, científica y política, acostumbrada a mirar las cosas desde otra perspectiva. Las políticas de desarrollo agrícola, las de inversión agropecuario y forestal, las de inversiones en agricultura y las C\&T, se aproximan a la realidad y a los destinatarios a partir de una visión y de instrumentos de intervención verticales, en general especializados por rubros de producción. Insistimos en este concepto porque aquí está la verdadera diferencia en el abordaje de los procesos de diseño, aplicación y evaluación de las políticas y la justificación a su carácter de "diferenciadas". La política diferenciada para la AF exigió en cambio - una mirada más sistémica u holística, puesto que el foco no era "la productividad o la competitividad del rubro", sino la productividad, la competitividad y la calidad de vida de la unidad productiva familiar. Para esto ni los Estado, ni la academia, ni los institutos de investigación, ni aún las agencias de cooperación estaban del todo preparados.

Durante la década de los 2000, quedaba una especie de "memoria histórica" de los llamados procesos de "Desarrollo Rural Integral" de la década de los 60 del siglo pasado, cuyas prácticas y modelos de intervención buscaron aplicar los resultados de la "Revolución Verde" a sectores de población rural denominados como aquellos "menos favorecidos" en la búsqueda de la competitividad económica, los más vulnerables o con alto riesgo de expulsión de los territorios, por el implacable proceso de concentración económica, intrínseco al modelo de la "Revolución Verde" y lo que éste implicó en la producción y el comercio agrícola y de alimentos.

Esta memora histórica no fue - ni es aún al momento de escribir este artículo suficiente como para asegurar la vigencia y la sostenibilidad de la AF como una forma de organización del trabajo rural y agrícola, y del desarrollo rural, de manera mucho más sostenible, inclusiva y económicamente eficaz.

Para que la afirmación anterior sea cierta y para que los Estados puedan modernizar su institucionalidad orientada a la inversión en el desarrollo agrícola y rural, los procesos de focalización (targeting), son claves para un mejor diseño de los instrumentos, lo cual permitirá aplicar mejor los recursos obteniendo:

(i) mejores resultados (cambio en las situaciones de subordinación y pobreza; adopción de buenas prácticas agrícolas y de organización del trabajo; adopción de tecnologías apropiadas para aumentar productividad, mejorar la seguridad alimentaria, el ingreso y la calidad de vida, etc);

(ii) mayor impacto (por unidad de recurso invertido, a partir de una mayor cobertura de la población destinataria de las políticas públicas y obtención de resultados en menor tiempo. Morigerar el riesgo de desvío de los recursos hacia otros sectores de la población no destinatarios)

Ocurre - y es común - registrar la siguientes situaciones en el campo: (a) muchas veces los instrumentos existen pero la forma, características de los mismos, así como los requisitos para acceder a ellos, hacen que gran parte de la población destinataria no los visualiza o identifica y/o no tienen condiciones reales de acceso, por lo cual los recursos se 
sub - utilizan, la situación queda inmodificada y se amplía la frustración de los actores involucrados; (b) los instrumentos son diseñados de tal forma (general o inespecífica) que terminan siendo utilizado y los recursos apropiados por otro tipo de productor o población diferente a los que inicialmente no fueron dirigidos. Son aquellos con mayores capacidades desarrolladas y que fácilmente tienen acceso a los mismos. Muchas veces frente a la sub utilización de recursos los propios agentes técnicos orientan los mismos hacia aquellos productores con mayores capacidades, esto en si mismo genera la reproducción de situaciones de subordinación que se quería cambiar.

Estos elementos solo para resaltar la importancia en una más "ajustada" definición de la categoría AF, un más "ajustado" diseño de los instrumentos y de la asignación de los recursos, tomando en cuenta: cantidad, duración o plazos, flujo y acceso a partir de una mejor y más precisa identificación de los destinatarios de los mismos.

\section{Evolución de la visibilidad de la AF para las políticas públicas}

Para disponer de más y mejores políticas públicas para la AF. Mejores diseños e instrumentos mejor aplicados y más recursos con mayor impacto no solo alcanza con las definiciones generales sobre la AF y los criterios que nos permiten llegar a ellos, sino que hay que encontrar parámetros concretos a los que se les pueda asignar valores. Valores diferentes y que obviamente reflejen y respeten las condiciones de contexto, geográfico, agroecológico - productivo, socio - cultural e histórico

El abordaje productivo y económico es necesario pero notoriamente insuficiente. Históricamente los instrumentos de "data collection" a nivel agropecuario - básicamente los censos - han tenido y tienen una orientación económica, productiva, tecnológica y en alguna menor medida social. Con ello es posible caracterizar con mucha fidelidad las peculiaridades del sector agropecuario (a nivel físico / económico / productivo), sus avances, estado de situación, comportamiento, dentro de él los diferentes sub - sectores y comportamientos por rubros de producción y así poder disponer de información estadística válida y fiable para el diseño de las políticas por rubro o sub - sector, o aún sectoriales.

No obstante el abordaje de la AF como una categoría particular que incorpora para su definición no solo el conocimiento proveniente características del área económica y/o de las ciencias biológicas, sino que para comprender la amplitud y heterogeneidad del concepto y de la casuística real y concreta en los territorios, implica la aplicación de otras áreas del conocimiento como la sociología, historia y antropología, entre otras y que son necesarias para el análisis y para una cabal comprensión del fenómeno, sus implicancias para la vida de la sociedad y para el desarrollo de los territorios rurales y en general el desarrollo equilibrado de los países. Disponer de una interpretación correcta de las implicancias de, la AF como categoría de análisis social y económico, de su papel y de sus implicancias relativas al desarrollo, implicaría disponer de información certera y fiable sobre ésta. Saber con certeza y en forma cuantitativa y cualitativa el comportamiento de los principales parámetros e indicadores que le caracterizan. Para ello las herramientas generalmente utilizadas para la recolección de datos en el medio rural, los censos agropecuarios y las encuestas, deberían incorporar otra categoría de indicadores y parámetros que permitan "poner valor" a los criterios aceptados.

Figura 1. "Círculo vicioso" que impide la visibilidad estadística de la AF, para tomar mejores decisiones: 

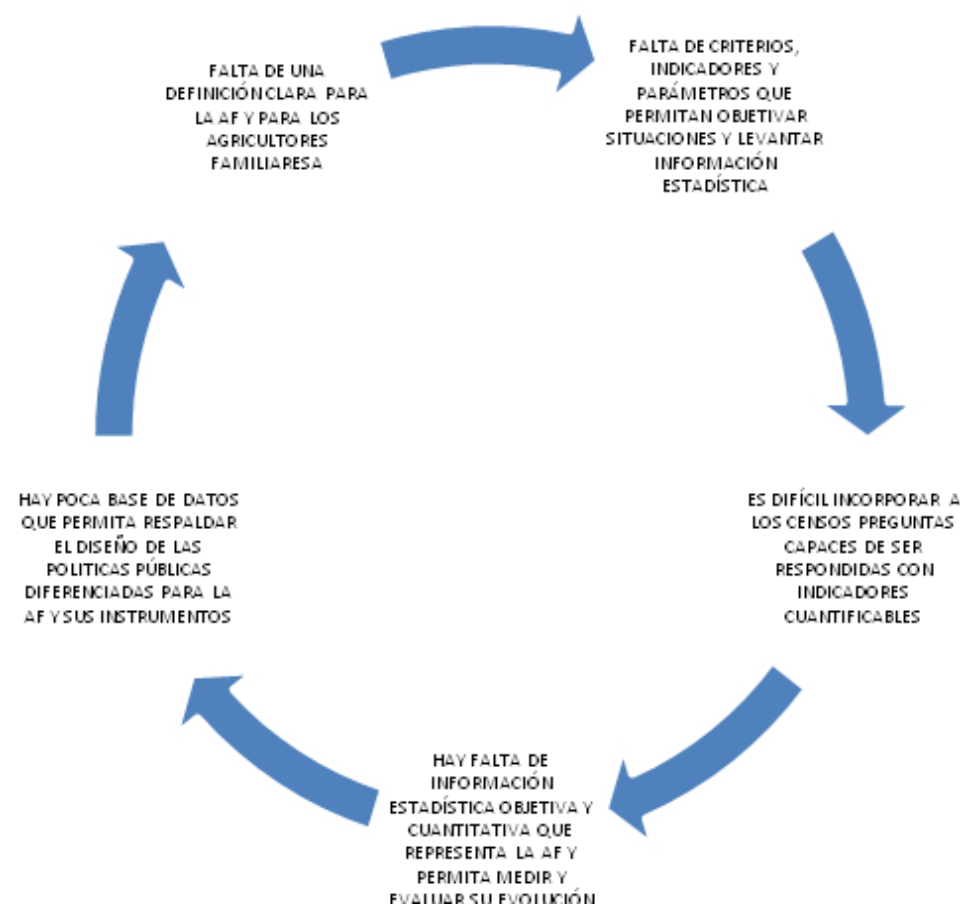

Lo anterior está altamente relacionado con la realidad de las políticas agropecuarias y/o las políticas para el desarrollo rural y más recientemente con la necesidad de mejorar la calidad y la focalización de las políticas diferenciadas para la AF

Cómo ya hemos señalado, desde la segunda mitad del siglo XX, pasando por la "Revolución Verde", por las políticas del Çonsenso de Washington" de los 90, las clásicas políticas agropecuarias se orientaron exclusivamente hacia los aumentos de productividad y competitividad económica de la producción agropecuaria y de alimentos. Recién a los inicios de la década de los 2000 aparece la AF como una categoría a diferenciar dentro de la "Agricultura" en general. Pero no fue una aparición fácil de visualizar ni de justificar conceptual y objetivamente. La existencia de "una sola agricultura" las políticas verticales hacían difícil que a nivel político y técnico se aceptara otra visión.

No obstante las necesidades de (i) intentar frenar el flujo migratorio de las áreas rurales a las urbanas; (ii) hacer un uso más racional de la tierra como factor de producción y como bien social, para producir una cantidad mayor de alimentos, preservando los recursos naturales y el ambiente; (iii) atender el desarrollo de los territorios, tomando el concepto de territorio como "una construcción social"; (iv) la necesidad de mejorar la eficiencia, eficacia y oportunidad con que los Estados llegan a los territorios y a la población y a los agricultores con los bienes y servicios públicos y las obras de infraestructura y que esto a su vez tiene impacto sobre la competitividad; (v) los desafíos de la Seguridad Alimentaria y Nutricional, no solo en las áreas rurales sino fundamentalmente en las zonas urbanas y el acceso a los alimentos por parte de la población más vulnerable; (vi) y finalmente el proceso de suba en el precio de las commodities agrícolas y en especial de los alimentos que - más allá de una volatilidad siempre presente - en los últimos 11 años se ha mantenido como tendencia y su impacto en las estrategias y políticas de Seguridad Alimentaria y Nutricional (SAN). Todo lo anterior sumado, ha permitido en los últimos 15 
Definición de “Agricultura Familiar” como categoría Socioeconómica

años una visibilización mayor de la AF como categoría socio económica y como destinataria de políticas públicas diferenciadas

Es necesario perfeccionar la forma cómo los instrumentos y los recursos de las políticas "bajan" a los territorios y son apropiados por los verdaderos destinatarios y cómo las agencias de cooperación internacional complementan capacidades, saberes y recursos para facilitar junto con los Gobiernos y las organizaciones sociales y productivas de los AF este proceso.

Quizás intentando interpretar algunas causas de la "invisibilidad de la AF" por décadas, se pueden encontrar razones en la necesidad de dar cuenta de una demanda creciente de alimentos en grandes cantidades y en plazos menores. La excesiva orientación competitiva a los mercados de las políticas tradicionales en algunos países, con un modelo productivo basado en el volumen y la acumulación de capital, tecnología y la aparición de otros agentes económicos de fuera del sector agrícola invirtiendo en esta modalidad productiva con tasas de rentabilidad muy atractivas y demandas en expansión con sostenibilidad, llevaron a privilegiar aún más las políticas "verticales" y las políticas por rubro o sub sector. Los sectores de población que no fueran capaces de acompañar este crecimiento competitivo por la vía de la acumulación, debían ser atendidos por políticas de carácter compensatorio, estimulándolos a diversificarse hacia otras producciones y/o a salir del sector y del medio rural (décadas de los 70 a los 90). El modelo concentrador eficiente en volumen estimulado por altos precios y rentabilidad, generó dificultades en la 'gobernanza' de los mercados de alimentos y si bien fue una solución para el aumento de las cantidades producidas generó grandes dificultades de acceso en relación a las buenas políticas de SAN (disponibilidad y acceso, calidad, dieta equilibrada).

Por otro lado en determinadas y muchas regiones del mundo más allá que el modelo económicamente dominante fuese la agricultura comercial, altamente concentrada, la gran mayoría de la población rural hace parte de la AF y por lo tanto hay una diferenciación implícita, que paradojalmente no hacía necesario explicitar la situación y características de la AF, más allá que en términos de diseño y aplicación de políticas e instrumentos, predominó y predomina la visión "vertical" y por rubro, y el mayor "talón de Aquiles" de los proceso de desarrollo es como llegan las políticas a los territorios y comunidades rurales. La lucha contra las causas de la pobreza rural y la SAN han sido un gran removedor en el aumento de la "visibilidad" publica y política de la AF y del cambio de paradigma en términos que la AF no solo no es parte del problema de la pobreza, sino que con políticas apropiadas y sostenidas es parte de la solución a la misma, como se demuestra en varias regiones del mundo, por ejemplo en ALC.

El desafío ahora es reconocer la heterogenidad de la AF y comenzar a hablar de "MATRICES DE POLÍTCAS PÚBLICAS" que permitan atender la complejidad de la finca familiar, la necesidad de asociar las mismas para asegurar el autoconsumo familiar y competir, así como la necesidad de asegurar los parámetros de la SAN.

El diagrama que de la página siguiente(Figura 2.), muestra como las políticas e instrumentos diferenciados para la $\mathrm{AF}$, se vinculan con otras áreas de la política pública que no dependen ni política ni presupuestalmente de los Ministerios Sectoriales de Agricultura y/o Desarrollo Rural, que incluso involucran diferentes niveles del Estado (Nacional, Estadual, Municipal), pero que son claves al momento de encarar los procesos de desarrollo de los agricultores y en general de la población rural y sus comunidades. Este razonamiento está dicho solo para afirmar la necesidad de: (i) definir con la mayor precisión posible a la AF y sus diferentes sub - categorías; (ii) facilitar su localización en los territorios; (iii) 
darles visibilidad estadística para focalizar mejor la aplicación de recursos, que hay que articular y complementar desde el Estado.

Así mismo la matriz que le sigue, "abre el menú" de políticas e instrumentos diferenciados y se presenta como una necesaria combinación de políticas e instrumentos al momento del abordaje de las realdades territoriales. Para aplicar las mismas implica una adecuación de la institucionalidad pública agrícola/ agropecuaria para la generación de bienes y servicios públicos y para definir los niveles y los tipos de inversión en los territorios, la participación de la sociedad civil rural organizada en la definición de la estrategias de desarrollo y asegurar que por esta vía que la demanda de alimentos podrá ser atendida de manera sostenible sin entrar en conflicto con otras formas de organización de la producción, sino complementándose con ellas, sin subordinación.

Figura 2. Diagrama que presenta la relación entre las políticas públicas diferenciadas para la af y otras áreas de la política pública directamente vinculadas

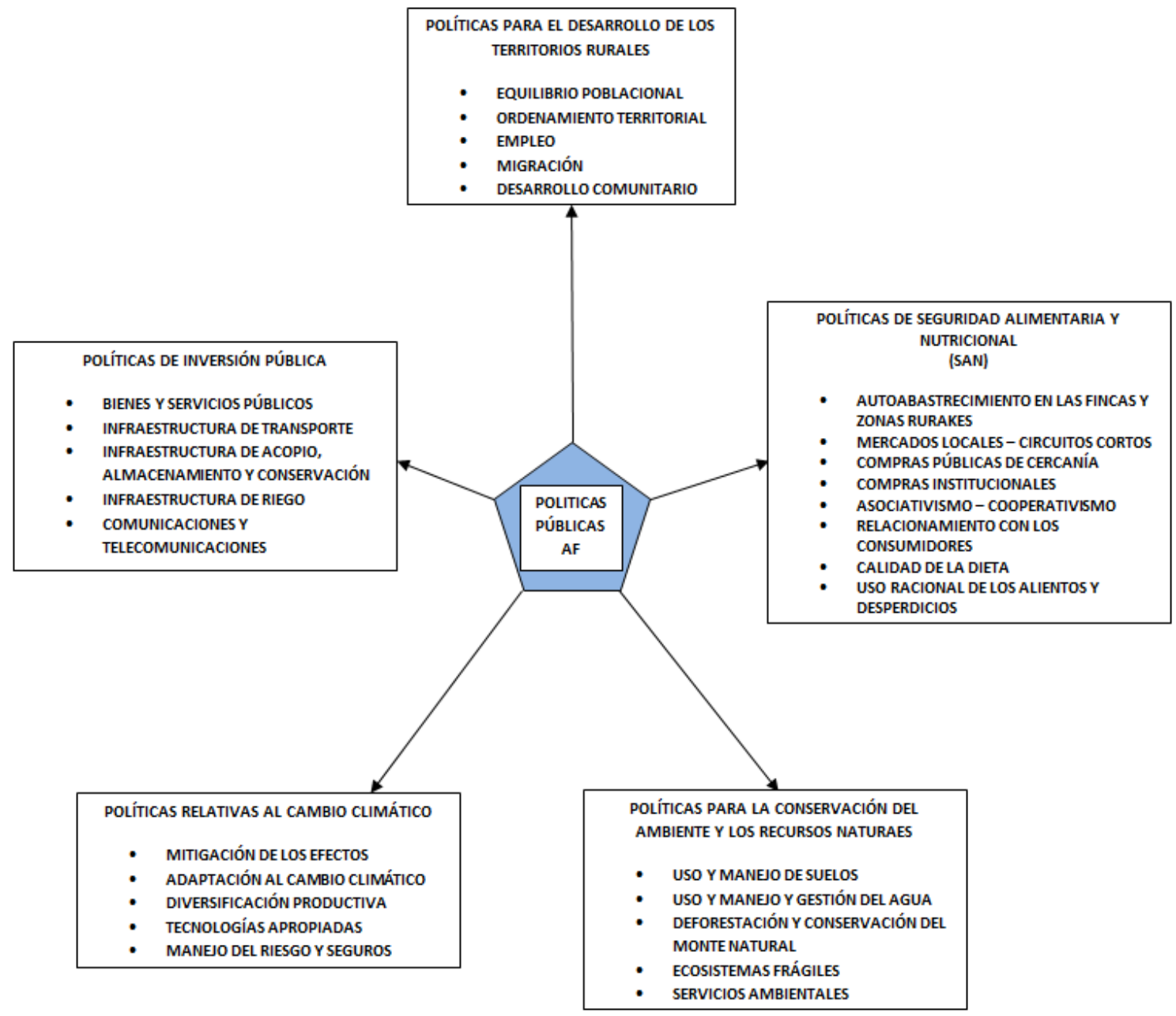




\begin{tabular}{|c|c|c|c|c|c|c|c|c|c|c|}
\hline $\begin{array}{l}\text { MATRIZ DE } \\
\text { POLÍTICAS E } \\
\text { INSTRUMEN- } \\
\text { TOS }\end{array}$ & $\begin{array}{l}\text { ACCESO Y } \\
\text { TENENCIA } \\
\text { A LA } \\
\text { TIERRA }\end{array}$ & $\begin{array}{l}\text { C\&T - } \\
\text { ASISTENCIA } \\
\text { TÉCNICA }\end{array}$ & CRÉDITO & $\begin{array}{l}\text { USO Y } \\
\text { MANESO } \\
\text { DE } \\
\text { SUELOS Y } \\
\text { AGUA }\end{array}$ & $\begin{array}{l}\text { SEGURO } \\
\text { AGRÍCOLA }\end{array}$ & $\begin{array}{l}\text { INVERSIONES } \\
\text { MULT - } \\
\text { PREDIALES }\end{array}$ & $\begin{array}{l}\text { RIEGO Y } \\
\text { DRENAJE }\end{array}$ & $\begin{array}{l}\text { PRECIOS Y } \\
\text { MERCADEO }\end{array}$ & $\begin{array}{l}\text { CADENAS DE } \\
\text { VALOR }\end{array}$ & $\begin{array}{l}\text { COMPRAS } \\
\text { PÚBLICAS }\end{array}$ \\
\hline $\begin{array}{l}\text { EXTENSIÓN } \\
\text { RURAL }\end{array}$ & $\begin{array}{l}\text { Reforma } \\
\text { Agraria Y } \\
\text { Servicios de } \\
\text { ATER }\end{array}$ & $\begin{array}{l}\text { C \& T } \\
\text { Apropiad as. } \\
\text { Semillas y } \\
\text { Varied ad es } \\
\text { Sistemas de } \\
\text { ATER }\end{array}$ & $\begin{array}{l}\text { Manejo del } \\
\text { crédito } \\
\text { Montos, } \\
\text { Destinos, } \\
\text { Plazos, } \\
\text { Tasas } \\
\text { Devolución }\end{array}$ & $\begin{array}{l}\text { ATER } \\
\text { Sistemas de } \\
\text { uso y man ejo }\end{array}$ & ATER & $\begin{array}{l}\text { Creación de } \\
\text { empresas y } \\
\text { cooperativas de } \\
\text { servicios y/o } \\
\text { producción en } \\
\text { común }\end{array}$ & $\begin{array}{l}\text { Diseño de } \\
\text { sistemas y } \\
\text { asistencia } \\
\text { técnica }\end{array}$ & $\begin{array}{l}\text { Fortalecimiento } \\
\text { de } \\
\text { organizaciones } \\
\text { Rurales } \\
\text { Gestión de la } \\
\text { Información }\end{array}$ & $\begin{array}{l}\text { ATER } \\
\text { Fortalecimiento } \\
\text { de } \\
\text { Organizaciones } \\
\text { Rurales }\end{array}$ & $\begin{array}{l}\text { ATER } \\
\text { Fortalecimient } \\
\text { O de } \\
\text { Organizaciones } \\
\text { Rurales }\end{array}$ \\
\hline $\begin{array}{l}\text { SERVICIOS } \\
\text { FINANCIEROS } \\
\text { RURALES }\end{array}$ & $\begin{array}{l}\text { Créditos } \\
\text { Fundiarios }\end{array}$ & $\begin{array}{l}\text { Crédito } \\
\text { Supervisado }\end{array}$ & $\begin{array}{l}\text { Equipos } \\
\text { Herramien- } \\
\text { tas elnv. } \\
\text { Físicas } \\
\text { Capital de } \\
\text { Trabajo }\end{array}$ & $\begin{array}{l}\text { Crédito } \\
\text { supervisado }\end{array}$ & $\begin{array}{l}\text { Subsidio de } \\
\text { primas }\end{array}$ & $\begin{array}{l}\text { Financiamiento a } \\
\text { organizaciones, } \\
\text { cooperativas y } \\
\text { empresas de } \\
\text { servicios } \\
\text { asociativas }\end{array}$ & $\begin{array}{l}\text { Crédito para } \\
\text { inversiones } \\
\text { en equipos, } \\
\text { represas y } \\
\text { pozos }\end{array}$ & $\begin{array}{l}\text { Adelantos sobre } \\
\text { laventa futura } \\
\text { dela producción }\end{array}$ & $\begin{array}{l}\text { Finan ciamiento } \\
\text { parala } \\
\text { integración de } \\
\text { las Cadena, en } \\
\text { diversos } \\
\text { eslabones }\end{array}$ & $\begin{array}{l}\text { Créditosy } \\
\text { ad elantos } \\
\text { sobrenegocios }\end{array}$ \\
\hline $\begin{array}{l}\text { ADAPTACIÓN } \\
\text { MImGACIÓN } \\
\text { ALCC }\end{array}$ & & $\begin{array}{l}\text { Tecnolog. } \\
\text { Apropiad as } \\
\text { Nu evas } \\
\text { Varied ad es }\end{array}$ & & $\begin{array}{l}\text { ATER y } \\
\text { nuevos } \\
\text { sistemas }\end{array}$ & $\begin{array}{l}\text { Gestión de } \\
\text { Riesgo }\end{array}$ & & & & & \\
\hline $\begin{array}{l}\text { BIENES Y } \\
\text { SERVICIOS } \\
\text { PÚBLIICOS }\end{array}$ & $\begin{array}{l}\text { REFORMA } \\
\text { AGRARIA }\end{array}$ & $\begin{array}{l}\text { Sistemas de } \\
\text { ATER }\end{array}$ & $\begin{array}{l}\text { Primas de } \\
\text { seguros } \\
\text { Agrícolas }\end{array}$ & $\begin{array}{l}\text { Inversones } \\
\text { en } \\
\text { conservación } \\
\text { sistemas y } \\
\text { uso desuelos }\end{array}$ & $\begin{array}{l}\text { Sistemas de } \\
\text { Alerta } \\
\text { Temprana }\end{array}$ & $\begin{array}{l}\text { Caminos Rurales } \\
\text { Redes Eléctricas } \\
\text { Comercio } \\
\text { Transportey } \\
\text { Logística } \\
\text { Salud } \\
\text { Educación }\end{array}$ & $\begin{array}{l}\text { Reservas } \\
\text { Alumbtramie } \\
\text { nto de Aguay } \\
\text { Canales de } \\
\text { Riego }\end{array}$ & $\begin{array}{l}\text { Sistemas de } \\
\text { Información } \\
\text { Manejo y acceso } \\
\text { a TIC's }\end{array}$ & $\begin{array}{l}\text { Infraestructura } \\
\text { pública de } \\
\text { caminos, } \\
\text { caden as de frío, } \\
\text { almacen aje }\end{array}$ & $\begin{array}{l}\text { Infraestructura } \\
\text { pública de } \\
\text { caminos, } \\
\text { cadenas de } \\
\text { frío, } \\
\text { almacen aje }\end{array}$ \\
\hline $\begin{array}{l}\text { ORGANIZA } \\
\text { CIONES } \\
\text { ASOCIATIVAS }\end{array}$ & $\begin{array}{l}\text { Explotación } \\
\text { en conjunto } \\
\text { dela tierra. } \\
\text { Campos de } \\
\text { pastoreo y } \\
\text { recría, y } \\
\text { semillas }\end{array}$ & $\begin{array}{l}\text { Cooperativas } \\
\text { deservicios de } \\
\text { ATER }\end{array}$ & $\begin{array}{l}\text { Coops. De } \\
\text { Ahorro y } \\
\text { Crédito y } \\
\text { Sistemas de } \\
\text { Micro } \\
\text { crédito }\end{array}$ & $\begin{array}{l}\text { Cooperativas } \\
\text { deuso dela } \\
\text { tierray de } \\
\text { regantes }\end{array}$ & $\begin{array}{l}\text { Seguros } \\
\text { Colectivos }\end{array}$ & $\begin{array}{l}\text { Equipamiento } \\
\text { multipredial de } \\
\text { maquinaria } \\
\text { agrícola }\end{array}$ & $\begin{array}{l}\text { Cooperativas } \\
\text { deregantes }\end{array}$ & $\begin{array}{l}\text { Empeesas } \\
\text { asociativas de } \\
\text { servicios a la } \\
\text { comercialización }\end{array}$ & $\begin{array}{l}\text { Cooperativas } \\
\text { intermedias de } \\
\text { agregado de } \\
\text { valor }\end{array}$ & $\begin{array}{l}\text { Cooperativas } \\
\text { intermedias }\end{array}$ \\
\hline $\begin{array}{l}\text { SEGURIDAD } \\
\text { SOCIAL ESTÍMUO } \\
\text { AL RETIRO Y AL } \\
\text { AFINCAMIENTO } \\
\text { DELA JUVENTUD } \\
\text { RURAL }\end{array}$ & $\begin{array}{l}\text { Monotributo } \\
\text { para } \\
\text { asentadosy } \\
\text { colonos }\end{array}$ & $\begin{array}{l}\text { ATER } \\
\text { Afincamiento } \\
\text { dejóvenes } \\
\text { rurales }\end{array}$ & $\begin{array}{l}\text { Créditos de } \\
\text { in iciación }\end{array}$ & & & $\begin{array}{l}\text { Créditos de } \\
\text { Iniciación }\end{array}$ & & & $\begin{array}{l}\text { Estímulo a } \\
\text { empresas de } \\
\text { jóvenes } \\
\text { Reorganización } \\
\text { del trabajo }\end{array}$ & $\begin{array}{l}\text { Estímulo a } \\
\text { empresas de } \\
\text { jóvenes } \\
\text { Reorganización } \\
\text { del trab ajo }\end{array}$ \\
\hline $\begin{array}{l}\text { EQUIDAD DE } \\
\text { GÉNERO }\end{array}$ & $\begin{array}{l}\text { Acceso, } \\
\text { titulación y } \\
\text { tenencia }\end{array}$ & ATER & $\begin{array}{l}\text { Reconoci- } \\
\text { miento y } \\
\text { acceso }\end{array}$ & & $\begin{array}{l}\text { Reconoci- } \\
\text { miento y } \\
\text { acceso }\end{array}$ & $\begin{array}{l}\text { Reconocnimento y } \\
\text { acceso }\end{array}$ & & & $\begin{array}{l}\text { Acceso } \\
\text { diferenciado a } \\
\text { latitularidad de } \\
\text { empresas }\end{array}$ & $\begin{array}{l}\text { Acceso } \\
\text { diferenciado a } \\
\text { latitularidad } \\
\text { de empresas }\end{array}$ \\
\hline
\end{tabular}




\section{La AF como categoría socioeconómica y/o de acumulación - una cuestión de enfoque}

En la realidad las dificultades que se han encontrado para establecer definiciones y conceptos comunes y para luego definir criterios, parámetros e indicadores cuyo relevamiento pueda tener validez estadística y permitir así una mejor focalización de las políticas y localización de los destinatarios, deriva que estamos trabajando con un concepto multidimensional, que involucra elementos de la dimensión económica, pero también de las dimensiones del conocimiento vinculadas a los aspectos sociales, culturales e históricos.

Se trata de identificar e interpretar el comportamiento de sectores de población que establecen con el territorio y más precisamente con la tierra y más precisamente aún, con el suelo, una relación que va mucho más allá del mercado. Que tiene que ver con raíces ancestrales, culturales y de arraigo personal y familiar. Se genera una "forma de vida" y desde allí una forma de interpretar la realidad y planificar el futuro. Por ello una interpretación solamente económica termina siendo excesivamente reduccionista, aunque puede generarnos la ilusión que llegamos a cierta interpretación de la realidad.

La discusión sobre el concepto de AF en los últimos 15 años involucra las dos áreas del conocimiento mencionadas: (a) la económica y (b) la socio / cultural / histórica. Una sola de las dos es incompleta para entender el fenómeno que implica la AF.

Finalmente, encarar una análisis de la AF como categoría socio / económica no es atemporal ni a - geográfica, está fuertemente vinculada a cada contexto y cada contexto tiene que ver con estos factores: (a) los derivados de la situación geográfica y agroecológica y distancia relativa a los mercados; (b) los derivados de los procesos económicos; (c) los derivados de la historia, la cultura, la relación con la tierra y el territorio.

No obstante no debemos perder de vista el objetivo del trabajo realizado por el Panel de Expertos Internacionales, este consistió en encontrar conceptos, criterios, parámetros e indicadores que permitan hacer una valoración objetiva de situaciones de la AF más allá de su heterogeneidad. El trabajo permitió mostrar "una especie de fotografía multifacética de la diversidad sub - regional, pero permita a la vez identificar con nitidez a los destinatarios de las políticas públicas"

Los trabajos revisados durante esta investigación indican que en las diferentes regiones del mundo, hay un acuerdo al menos en los niveles "académico" y de representación social, de referir a la AGRICULTURA FAMILIAR, como un "concepto paraguas", debajo del cual existen diferentes sub - categorías que permiten ajustar la definición y vincularla con sus respectivos contextos, sin hacerles perder consistencia con la definición mayor. Por ejemplo la definición adoptada por $\mathrm{FAO}^{1}$.

\section{${ }^{1}$ DEFINICIÓN DE FAO SOBRE AF}

Se entiende por Agricultura Familiar a la producción agrícola, pecuaria, forestal, pesquera y acuícola que, pese a su gran heterogeneidad entre países y al interior de cada país, posee las siguientes características principales: (i) acceso limitado a recursos de tierra y capital; (ii) uso preponderante de fuerza de trabajo familiar, siendo el(la) jefe(a) de familia quien participa de manera directa del proceso productivo; es decir, aún cuando pueda existir cierta división del trabajo, el(la) jefe(a) de familia no asume funciones exclusivas de gerente, sino que es un trabajador más del núcleo familiar; (iii) la actividad agropecuaria/silvícola/pesquera/acuícola es la principal fuente de ingresos del núcleo familiar, que puede ser complementada con otras actividades no agrícolas que se realizan dentro o fuera de la unidad familiar (servicios relacionados con el turismo rural, beneficios ambientales, producción artesanal, pequeñas agroindustrias, empleos ocasionales, etc.). 
De acuerdo a la experiencia revisada y a la bibliografía consultada y las respuestas obtenidas de cerca de una veintena de expertos, participantes en el Panel Internacional de Expertos, la propia definición general y las sub - categorías deben de alguna manera tomar en cuenta los siguientes criterios (los mayoritariamente mencionados, tanto en la bibliografía como por los expertos consultados): (a) relación entre el trabajo familiar y el trabajo contratado externo a la familia; (b) la administración de la finca; es decir quién toma las decisiones principales; (c) sistemas productivos diversificados y claramente alejados de la "monocultura" y (d) orientación de la actividad productiva familiar hacia el autoconsumo de los productos de la finca o hacia los mercados.

Por lo tanto partiendo de la definición general de la FAO sobre la AF y tomando en cuenta los aportes de los expertos y participantes en el Panel Internacional de Expertos, llegamos a una definición "paraguas" y a siete sub - categorías representativas de diferentes realidades y contextos regionales y sociales:

Figura 3. Criterios generalmente aceptados a nivel internacional para definir $\mathrm{AF}$

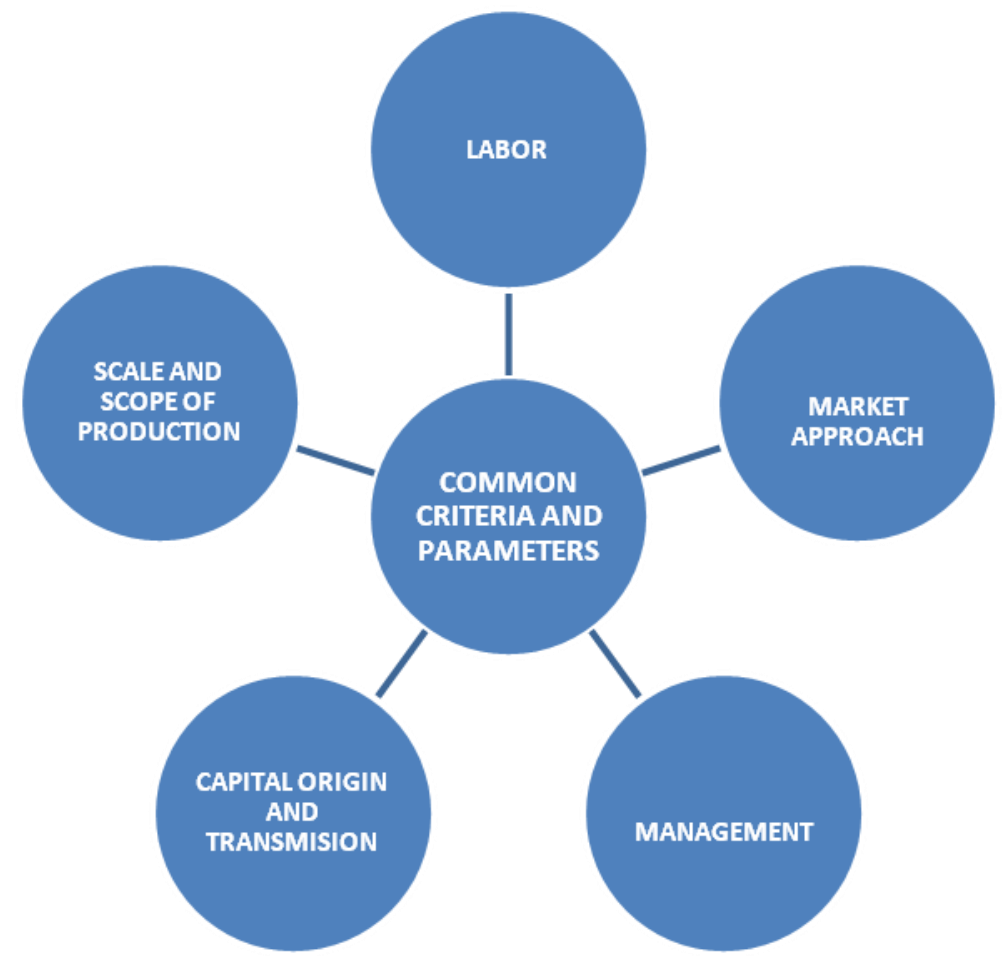

También para cada en cada uno de los criterios se encuentran "gradientes" en las situaciones, como puede verse en la siguiente caracterización de la AF.

i. AF con su producción claramente orientadas hacia los mercados; con acceso fluido a los diferentes mercados y a los factores de producción; capitalizados; asociados a organizaciones sociales y productivas de la AF, así como otras de servicios y cooperativas. La familia organiza el trabajo en la finca, contrata mano de obra en forma temporaria y/o contrata servicios específicos como laboreo de tierra u otros. Los ingresos provienen mayoritariamente de las actividades productivas de la finca. Se ubican sobre la línea de pobreza. 
ii. AF semi - capitalizados, con dificultades de acceso a algunos mercados como el financiero y factores de producción, principalmente acceso y tenencia de la tierra. Acceso a los mercados de productos a nivel local, estado de asociación preliminar o débil en organizaciones locales. La familia organiza el trabajo en el predio y toma las decisiones del negocio y la producción. En general sobre la línea de pobreza pero a veces con ingresos insuficientes, para atender las necesidades de liquidez de la familia para la compra de bienes y servicios. Gran parte de los alimentos que consumen lo obtienen del predio aunque en general vuelcan a los mercados excedentes de la producción y/o tienen un rubro de renta orientado al mercado.

iii. AF de subsistencia con dificultades de acceso y tenencia de la tierra, y ausencia de capital y grandes dificultades de acceso al crédito formal, bajo o nulo nivel de asociación, bajo la línea de pobreza. Básicamente con su producción orientada al autoconsumo. En general la organización del trabajo es muy precaria dentro de la finca, más allá que la familia toma las decisiones. Venden su fuerza de trabajo fuera de la finca, en general el jefe de familia) para poder obtener dinero con el cual comprar servicios esenciales. Pueden en ocasiones llevar excedentes de producción a mercados locales. Pueden recibir ayudas del Estado desde las políticas sociales y las productivas.

iv. AF de subsistencia que no llegan a cubrir sus necesidades alimentarias, tenencia irregular de la tierra, venden su fuerza de trabajo fuera de la finca, reciben ayuda del estado desde las políticas sociales y productivas. El trabajo familiar se reparte fuera de la finca y dentro de ella. Bajo la línea de pobreza.

v. Trabajadores Rurales, comunidades Agrícolas y comunidades aborígenes - pueblos originarios - comunidades raciales, pastores, pastores trashumantes y recolectores.

Presentadas en un diagrama simple las cinco categorías, se distribuirían espacialmente como se muestra en la figura siguiente. Las flechas en rojo son los flujos de intercambio y/o evolución de las familias entre una y otra sub - categoría, ya que tampoco las mismas son estáticas y hay un gradiente se situaciones entre ellas.

Figura 3. Diagrama de distibución de sub - categorías de la af de acuerdo a su orientación

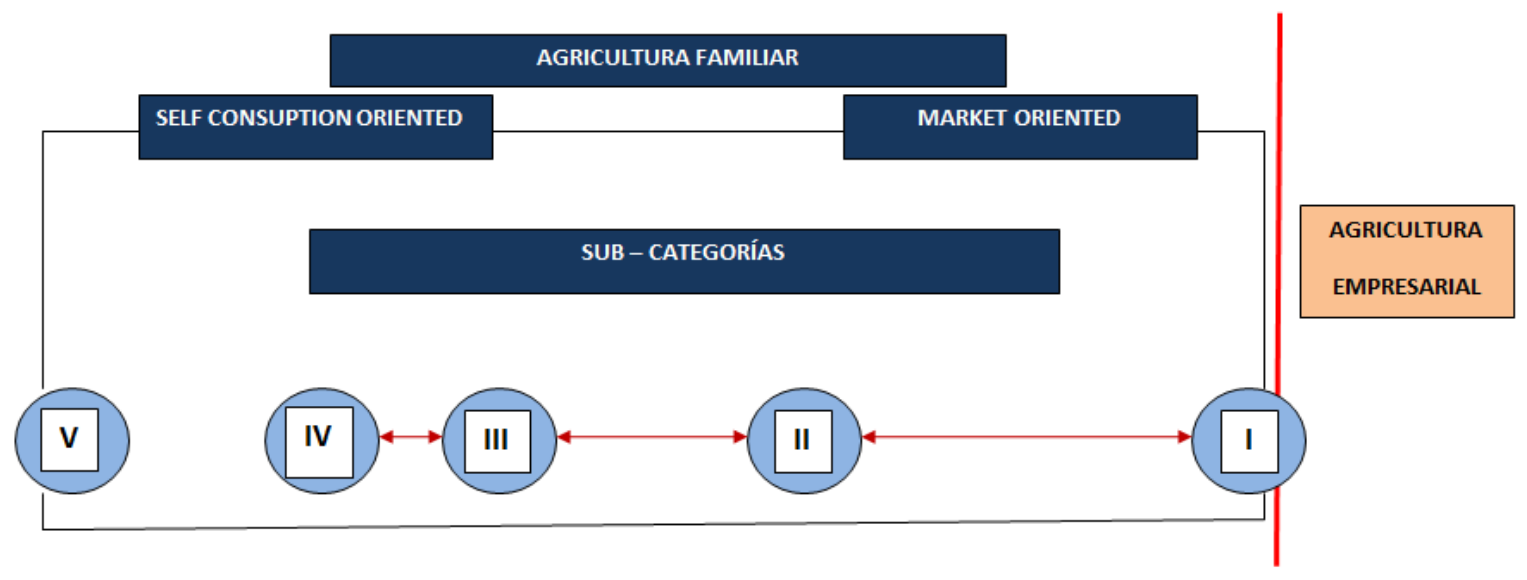




\section{Orientaciones o para encontrar definiciones compartidas y comparables sobre AF, a nivel internacional y que aseguren su "visibilidad estadística"}

Uno de los mayores logros del AIAF - 2014, ha sido el nivel de visibilidad pública y política ganada por la AF a nivel internacional, que se expresa en el reconocimiento indiscutible de su importancia como sector motor del desarrollo económico y social del medio rural.

No obstante lo anterior, existen aún obstáculos a superar vinculados a la variabilidad / heterogeneidad que la AF muestra, de acuerdo a diferentes contextos de desarrollo y geográficos. Esto es importante al momento de asegurar un tratamiento especial y diferenciado en las políticas e instrumentos y aún en el comercio. No se trata de modificar forzadamente la realidad para poder explicarla, sino que por el contrario se trata de aprender de ella y para ello, aplicar instrumentos de análisis objetivos y rigurosos.

El International Steering Committee (ISC) establecido en FAO para ejecutar los mandatos de la Asamblea General de las Naciones Unidas (UNAG), continuó trabajando más allá del AIAF 2014, para delinear los principios comunes que servirán como definición de la $\mathrm{AF}$, siendo que tanto en las acciones encaradas por el ISC, como en los trabajos técnicos realizados por el panel de expertos, se encontró con claridad que la AF es por lejos la forma dominante de agricultura en el mundo. De esta forma, se vió como muy recomendable continuar y profundizar este proceso, iniciado en 2014, y convocar a: (a) dirigentes sociales; (b) funcionarios de gobiernos; (c) académicos y científicos, a los efectos de encarar un abordaje técnico y científico de la visibilidad estadística de la AF y la consolidación de las políticas públicas diferenciadas, más allá de los avatares del contexto comercial, económico / financiero y político.

Para ello es necesario aumentar el conocimiento de la realidad y desarrollar la suficiente claridad conceptual para decidir cuál y cómo recoger la información clave, que sea estadísticamente válida para identificar y localizar a los agricultores familiares en los diversos contextos y territorios.

A partir de los mecanismos de consulta y la metodología participativa utilizada en el panel de expertos, se buscó llegar a definiciones y directrices para encarar los procesos a nivel nacional y regional que permita: (i) llegar a caracterizar la AF como una categoría socio / económica de organización de la producción agrícola; (ii) ajustar la misma mediante parámetros e indicadores sobre los criterios que permitan reconocer sub - categorías resultantes de la diversidad de la AF como una cualidad intrínseca a esta categoría; (iii) identificar con claridad y localizar en los territorios a los destinatarios de las políticas públicas diferenciadas para la AF; (iv) dar visibilidad estadística a la AF y montar mecanismos de registro, útiles para focalizar mejor las políticas, sus instrumentos y la asignación de recursos públicos para su desarrollo.

Las "Directrices" para un proceso de mejora de la calidad en el diseño y en la focalización de las políticas públicas diferenciadas para la AF, deberían contemplar un concepto $\boldsymbol{F L E X I B L E} \boldsymbol{E}$ INTEGRAL de la $\mathrm{AF}$ y un proceso que legitime las mismas:

(i) Tener claro los objetivos para lo cual se busca llegar a una definición general de AF y a determinadas sub - categorías en relación a diversos 
“contextos" geográficos, agroecológicos / productivo, social y cultural. No es una tarea de tipo académico ni teórica, sino que busca arribar a definiciones de carácter político, con criterios generales a los que luego puedan asignarse "parámetros" particulares para cuantificar los criterios y complementarlos con indicadores que objetiven situaciones particulares en un país, región y/o territorio de significación. Esta afirmación es significativa al momento de orientar los trabajos y especialmente los debates sobre la necesidad de llegar a "definiciones consensuadas". En última instancia siempre la decisión dependerá de una valoración política de cuanto la definición propuesta es representativa de la categoría y/o de las sub - categorías en relación a cada uno de los contextos.

(ii) Es una tarea que se realiza para mejorar la calidad en el diseño de las políticas públicas y sus instrumentos, por lo tanto debe significar "un compromiso" entre objetividad de los conceptos, la evidencia que los respalde y flexibilidad para no distorsionar la realidad a partir de necesidades analíticas;

(iii) Permitirá mejores posibilidades de "focalización" de las políticas, de sus instrumentos y de la asignación de los recursos, como así también (muy importante) de los flujos de llegada de los recursos a los destinatarios, de ahí que la vinculación en la definición y caracterización de las sub - categorías esté altamente relacionada con las realidades territoriales.

(iv) Permitirá reducir la brecha entre instrumentos y recursos existentes y disponibles, frente a destinatarios que no llegan a ellos por dificultades de información, capacidades organización y/o diseño;

(v) Es un proceso que requiere la participación de los actores de la sociedad civil, es decir las organizaciones que representan a los destinatarios y que el gobierno sabrá cuales son y deberá convocar en instancias o en "plataformas" de Diálogo Político;

(vi) Definidos los criterios para la Categoría General que permite llegar a una "categoría amplia" (umbrela) de AF, el siguiente paso será, encontrar los criterios específicos, parámetros e indicadores que permiten llegar a definir "sub - categorías";

(vii) Ajustar las sub - categorías a partir de un análisis compartido con las organizaciones sociales representativas de la AF en la plataforma de Dialogo Político (DP).

(viii) Crear un "Registro nacional y Voluntario de la AF" para ser destinatario de los instrumentos y recursos derivados de las políticas públicas para la AF.

(ix) Actualizar en forma periódica (mínimo cada tres años, máximo cada cinco) la base de datos del Registro Nacional.

(x) Incorporar a la brevedad en los instrumentos de recolección de datos (censos y encuestas) un sector o capítulo de la información a relevar vinculada a la AF como categoría y en función de los criterios consensuados. Llegar a definir entonces parámetros e indicadores que permitan caracterizar a las unidades familiares como tales, al menos en las categorías claves para su definición: (i) Organización del Trabajo; (ii) Gestión del predio; (iii) Origen del capital y forma de transmisión generacional; (iv) escala y orientación para los rubros principales de producción; (v) Grado de Vinculación con los mercados en relación al autoconsumo u orientación hacia diferentes tipo de mercado. 
(xi) Perfeccionar los mecanismos de recolección de información de carácter cualitativo, para ampliar y profundizar información sobre la AF que se puede incorporar como "secciones específicas" en censos y encuestas.

(xii) Control Social sobre la gestión, fidelidad y actualización de los Registros Nacionales, a partir de una participación y gestión de los Registros en forma compartida entre Estado / Organizaciones, cuando las condiciones del contexto lo habiliten. (Capital Social y Representatividad)

Esta propuesta orientadora permitiría generar una mayor "visibilidad política y estadística "de la AF en diferentes contextos nacionales y regionales. Su contenido surgió de las discusiones del Panel de Expertos, los aportes de sus participantes y del análisis de documentos de los organismos internacionales que han trabajado sobre esta materia en especial la FAO, el IFAD, el WAW y de organizaciones sociales representativas como La Vía Campesina, ROPA a nivel del África, CPROFAM a nivel de ALC y el World Rural Forum, que a su vez han volcado al diálogo los resultados de los debates en los más de 50 Comités Nacionales que se crearon y funcionaron en el marco del AIAF.

\section{Conclusiones}

El Concepto de AF es una construcción multidimensional. Dentro de ella los criterios fundamentales son: (i) organización del trabajo familiar en la finca y contratación eventual de mano de obra asalariada; (ii) administración de la finca o empresa familiar, o sea quién toma las decisiones productivas y/o técnicas y/o comerciales; (c) situación relativa de autonomía o dependencia respecto de los distintos mercados (insumos, factores, productos).

La dinámica de los cambios y su impacto sobre la agricultura en general, la AF y la población rural en distintos aspectos como: (i) los resultados de la revolución C\&T; (ii) los movimientos migratorios; (iii) las consecuencias de las movilizaciones y reivindicaciones sociales y de equidad de género; (iv) las condiciones políticas y de ciudadanía de la población rural; (v) la gobernanza de los mercados, principalmente de alimentos y su impacto sobre políticas y estrategias de SAN; (vi) los efectos del cambio climático, todo ello provoca transformaciones significativas en la actividad de millones de familias de agricultores, por lo tanto será muy difícil llegar a una única, comprensiva e inamovible definición de AF que pueda englobar todos estos aspectos y su heterogeneidad.

No obstante los criterios ya discutidos y generalmente aceptados, sí son de recibo para ser aplicados en distintas situaciones y contextos, y ayudan a seleccionar parámetros e indicadores, así como coeficientes, que permiten asignar valores (cuantificar) con validez estadística para objetivar realidades complejas y así focalizar mejor la definición de políticas e instrumentos y asignar recursos.

La aplicación de directrices del tipo de las propuestas desarrolladas más arriba en este texto, representará uno de los logros del AIAF en relación a la consolidación de la AF como el modelo de organización de la producción y el trabajo, más relevante en el sector agrícola a nivel mundial.

Como colofón nos gustaría dejar a modo de reflexión y los desafíos intrínsecos que ésta reflexión conlleva, la frase del Profesor Ye Jingzhong de la República Popular de China, quien expresaba: "el concepto de AF está en cambio permanente de la mano de los diferentes períodos de desarrollo y las diferentes fuerzas sociales". 


\section{BIBLIOGRAFÍA}

BÉLIÈRES et al.: Family Farming to deal with the challenges of sustainable development. "Family farming, to deal with the challenges of sustainable development (executive summary)", CIRAD, Agricultural Research for Development. 2014.

Disponible en: <http://www.cirad.fr/en/publications-resources/publishing/studies-anddocuments/les-agricultures-familiales-du-monde>

BÉLIÈRES et al.: Les agricultures familiales du monde. Définitions, contributions et politiques publiques. 2014. "Les agricultures familiales du monde", CIRAD, Agricultural Research for Development.

Disponible en: <http://www.cirad.fr/publications-ressources/edition/etudes-etdocuments/les-agricultures-familiales-du-monde>

DAVIDOVA, Sophia and THOMSON, Ken: "Family Farming: a Europe and Central Asia Perspective" Background Report for Regional Dialogue on Family Farming: Working towards a strategic approach to promote food security and nutrition. Brussels, 2013.

Disponible en:

<http://www.fao.org/fileadmin/user_upload/Europe/documents/Events_2013/FF_EUCAP_ en.pdf>

EDELMAN, Marc: "What is a peasant? What are peasantries?. Intergovernmental Working Group on a United Nations Declaration on the Rights of Peasants and Other People Working in Rural Areas. Geneva, 2013

EU Agricultural Economics Briefs. "Structure and dynamics of EU farms: changes, trends and policy relevance", No 9, October 2013.

<http://ec.europa.eu/agriculture/rural-area-economics/briefs/pdf/09_en.pdf>

European Commission Eurostat. "Glossary: Farm labour force - family labour”. Disponible en:

<http://epp.eurostat.ec.europa.eu/statistics_explained/index.php/Glossary:Farm_labour_for ce_-_family_labour>

FAO, "Agricultura Familiar en América Latina y el Caribe, Recomendaciones de Políticas". 2014. http://www.fao.org/publications/card/en/c/0236bc3c-b005-4def-9c42b9f509bcd2dc/

GRAUB, Benjamin; HERREN, Barbara; Wittman Hannah and Chappell, Jahi: "The state of Family Farmers in the World: global contributions and local insights for food and nutrition security and sustainable development". FAO, 2014

MACDONALD, James: "Family Farming in the United States". United States Department of Agriculture, Economic Research Service, 2014.

Disponible en: <http://www.ers.usda.gov/amber-waves/2014-march/family-farming-in-theunited-states.aspx\#.VBX011 fgWHl> 
RIMISP, IFAD. "La agricultura familiar en América Latina. Un nuevo análisis comparativo". 2014

SOURISSEAU, Jean-Michel: “Agricultures familiales et mondes à venir”. Editions Quae, 2014

World Agricultures Watch. "Towards an International Typology of Agricultural Holdings". Disponible en: http://www.worldagricultureswatch.org/content/towards-internationaltypology-agricultural-holdings

WORLD AGRICULTURES WATCH. "WAW proposed recommendations for WCA 2020", Working document February 2014.

"Alimentación escolar y las posibilidades de compra directa de la agricultura familiar. Estudio de caso en ocho países." Cooperación Brasil-FAO, Fortalecimiento de Programas de Alimentación Escolar en el Marco de la Iniciativa América Latina y Caribe Sin Hambre 2025, Proyecto GCP/RLA/180/BRA. Disponible en: 〈http://www.fao.org/docrep/018/i3413s/i3413s.pdf>

“A New Deal for Family Farmers", Asia Pacific Regional Consultation. Role of Family Farming in the 21stCentury: Achieving the Zero Hunger Challenge by 2025. Recommendations of the Chennai Consultation

"LEGACY of IYFF 2014 and the WAY FORWARD", Closing Ceremony of the AIAF 2014, Philippines, November $27^{\text {th }}$., 2014

"Perspectivas de la agricultura y del desarrollo rural en las Américas: una mirada hacia América Latina y el Caribe 2014". CEPAL, IICA y FAO 2014.

Disponible en: 〈http://www.fao.org/docrep/019/13702s/i3702s.pdf>

Submetido em 02/08/2016

Aprovado em 26/08/2016

Sobre o autor

Álvaro Ramos

Coordenador do Programa FIDA MERCOSUR .

E-mail: comunicacion@ fidamercosur.org 